\title{
LA POLÍTICA EXTERIOR COMÚN EN EL PROYECTO DETRATADO QUE INSTITUYE UNA CONSTITUCIÓN PARA EUROPA
}

FRANCISCO ALDECOA LUZARRAGA

Catedrático de Relaciones Internacionales

Cátedra Jean Monnet

Decano de la Facultad de Ciencias Políticas

y Sociología, Universidad Complutense de Madrid 


\section{SUMARIO}

1. La UNIÓN EuRopea ANTE LOS DESAFÍOS DEL NUEVO ENTORNO INTERNACIONAL: DOS debates Que CONVERgen.- 2. El debate sobre Política Exterior en la Convención EUROPEA.- 3 LA CONSTITUCIÓN EUROPEA Y SU MODELO POLITICO.- 4. UNIDADY COHERENCIA EN LA POLITICA EXTERIOR COMÚN.- 5. DEL INTERÉS NACIONAL A LA POLITICA DE responsabilidad.- 6. El Ministro de Asuntos Exteriores: la cara de la Política Exterior Común.- 7. La toma de decisiones en La Politica EXterior Y de Seguridad Común.- 8. Pasos hacia la Diplomacia Común Europea en la Constitución.- 9 la Politica Europea de Seguridad y Defensa: la geometría variable.- 10. La Política EXterior Común: expresión del federalismo interguBERNAMENTAL. 


\title{
LA POLÍTICA COMÚN EN EL PROYECTO DE TRATADO QUE INSTITUYE UNA CONSTITUCIÓN PARA EUROPA
}

\author{
POR \\ FRANCISCO ALDECOA LUZARRAGA \\ Catedrático de Relaciones Internacionales \\ Cátedra Jean Monnet \\ Decano de la Facultad de Ciencias Políticas \\ y Sociología, Universidad Complutense de Madrid
}

\section{LA UNIÓN EUROPEA ANTE LOS DESAFÍOS DEL NUEVO ENTORNO INTERNACIONAL: DOS DEBATES QUE CONVERGEN}

La Unión Europea es un actor internacional que ha sufrido una rápida evolución en los últimos años, pasando de tener una presencia internacional meramente económica a jugar cada vez más papeles políticos $y$ diplomáticos. "Esta transformación de actor comercial y económico a gran actor político y en el ámbito de la seguridad ha pasado en gran parte desapercibida" '. Precisamente cuando van a cumplirse diez años de la entrada en vigor del Tratado de Maastricht por el que se crea la Unión Europea de naturaleza política y su instrumento exterior, la Política

1 Javier SOLANA, "Multilateralismo eficaz: una estrategia para la UE", Política Exterior, $n^{\circ} 95$, sept-oct 2003 p. 37. 
Exterior Común, se perciben demandas crecientes de protagonismo de la Unión en la sociedad internacional de la globalización ${ }^{2}$.

Demandas que la Declaración de Laeken ha identificado, por lo que encargó a la Convención Europea que estudie las reformas necesarias para que la Unión pueda convertirse en un "factor de estabilidad" en el nuevo entorno globalizado y en "líder de numerosos paises y pueblos» ${ }^{3}$. Esas nuevas responsabilidades de la Unión provienen de diversos factores: la Unión que en 2004 reunirá a 450 millones de habitantes y producirá un cuarto del Producto Nacional Bruto mundial no puede vivir de espaldas a la escena internacional. De la misma manera, la propia ciudadanía europea, consciente de esta realidad, solicita de la Unión más presencia activa en la sociedad internacional4.

El propio factor internacional no es ajeno a la necesidad de profundización que experimenta la construcción europea en estos dias ${ }^{5}$. En este sentido, puede sostenerse que el 9 de noviembre de 1989 - caida del Muro de Berlín-y el 11 de septiembre de 2001 son fechas paradigmáticas comparables en alguna medida, ya que suponen cambios significativos en las relaciones internacionales. Ambas tienen repercusiones de primera magnitud en la construcción europea $y$, si generalmente se sostiene que el Tratado de Maastricht es hijo de los cambios en Europa y en el mundo puestos de manifiesto por el 9 de noviembre de 1989, no resulta menos cierto que el Tratado Constitucional es la respuesta europea al nuevo entorno internacional explicitado tras el 11 de Septiembre.

2 En este sentido, el Alto Representante para la PESC, Javier Solana recientemente afirmaba que "(...) Europa debe, puesm adaptarse y constituir uno de los puntales de la organización de un nuevo mundo más solidario y libre, más justo y seguro, del mundo que, al fin y al cabo, está en el alma del proyecto europeo". Javier SOLANA, Discurso en la reunión conjunta de las Comisiones de Asuntos Exteriores y Defensa de los Parlamentos nacionales de la Unión Europea, Bruselas, 10 de septiembre de 2003, p. 1, en: http://ue.eu.int.

3 Declaración de Laeken sobre el futuro de Europa, Conclusiones de la Presidencia, Consejo Europeo de Laeken, 14 y 15 de diciembre de 2001, en: http://ue.eu.int/summ.

4 En el mes de mayo de 2003 se han hecho públicos unos sondeos entre los ciudadanos de los Estados miembros sobre su postura en relación a una política exterior común europea y una política europea de defensa, pedidos por el Presidente Giscard a la Comisión. El $63 \%$ de los encuestados se declararon a favor de una política exterior europea mientras que el $71 \%$ quieren una política común de defensa. Honor MAHONY, “EU citizens want common defence and foreign policy", EUOberver, 5-5-2003.

5 En relación a la incidencia del factor internacional en el debate sobre el futuro y la finalidad de Europa véase: Francisco ALDECOA LUZARRAGA, "Una Europa": su proceso constituyente, Madrid, Bibliotecanueva, 2003, pp. 95-118. 
La Convención Europea y el Tratado Constitucional, sin embargo, no son los únicos cauces por los que se ha dirigido el debate sobre el papel de Europa en el mundo. En este sentido, el Consejo Europeo encargaba al Alto Representante para la PESC, Solana, la formulación de una Estrategia de Seguridad para Europa cuyo primer informe se presentaba al Consejo Europeo de Tesalónica de junio de 20036. Aunque no exista un vínculo directo o orgánico entre la Estrategia de Seguridad y la reforma constitucional «es evidente que constituyen las dos caras, una política y otra institucional, de una misma realidad ¿qué papel deseamos para qué Europa? La definición de un concepto estratégico implica identificar nuestros objetivos políticos, nuestro marco doctrinal común. La CIG y la nueva Constitución deberán aportarnos los medios institucionales para satisfacer las descritas ambiciones»?.

El informe presentado por Solana resulta inseparable de la Constitución, bebiendo ambos de una misma concepción del papel de Europa en la sociedad internacional de la globalización ${ }^{8}$. En el informe Solana se identifican las amenazas ante las que se encuentra el nuevo siglo (terrorismo, la proliferación de armas de destrucción masiva y la descomposición de Estados que vienen a ser sustituidos por la delincuencia organizada) y otras causas de inestabilidad como el hambre, el mal gobierno, el cambio climático o la dependencia energética.

El documento esboza las "recetas" para los males que asolan el mundo, recetas cuyos principios activos se inspiran en la recién nacida Constitución: la responsabilidad europea ante una globalización más justa, el imperio y desarrollo del Derecho internacional, la consolidación de Naciones Unidas, la protección de los derechos humanos, el multilateralismo, el avance hacia instituciones internacionales fuertes, la promoción de marcos regionales y las relaciones con otros actores aunando fuerzas. En definitiva, lo que se persigue es la regulación internacional como modelo de gestión del planeta.

6 Javie SOLANA, Una Europa en un mundo mejor, Informe al Consejo Europeo de Tesalónica, 20 de junio de 2003, en: http://ue.eu.int.

7 Javier SOLANA, Discurso en la reunión de las Comisiones de Asuntos Exteriores y Defensa de los Parlamentos nacionales de la Unión Europea, op. cit., p.5.

8 Distintos analistas así como los medios de comunicación insisten en subrayar que la Estrategia de Seguridad europea es una copia fiel de la norteamericana, siendo a nuestro juicio todo lo contrario, respondiendo a una visión típicamente europea de las relaciones internacionales. Coinciden con nuestro punto de vista: Steven EVERTS y Daniel KEOHANE, The European Convention and EU Foreign Policy: Learning from Failure, Centre for European Reform, 2 September 2003, p. 11, en: http://www.cer.org.uk. 
Los objetivos de la nueva estrategia europea persiguen fomentar la estabilidad en el entorno próximo a través del desarrollo de una política de vecindad que conste de relaciones privilegiadas, figura que por cierto también es objeto de un título en la Constitución. De forma más general se persigue construir un orden internacional basado en el multilateralismo efectivo cuyo marco fundamental ha de seguir siendo las Naciones Unidas. Por último, se busca adoptar un enfoque proactivo de cara a las amenazas, utilizando todos los medios que la Unión tiene a su alcance, políticos, económicos, comerciales, civiles, e incluso los militares.

\section{EL DEBATE SOBRE POLÍTICA EXTERIOR EN LA CONVENCIÓN EUROPEA}

El debate sobre el papel de Europa en el mundo y la reforma de la Política Exterior Común en el seno de la Convención ha sido uno de los más complejos. Es quizás una de las áreas más trabajadas, que más expectativas habia levantado y que más vaivenes ha sufrido durante los trabajos de la Convención. La política exterior y la defensa son ámbitos que pertenecen tradicionalmente al núcleo duro de los Estados y donde éstos son más reticentes a compartir soberanía. Además en el curso de los trabajos de la Convención se entrecruzó la cuestión de la guerra de Iraq, que dividió a los Estados europeos, en lo que Landáburu califica de "la mayor crisis en la historia de la construcción europea»?.

Desde las primeras sesiones de audiencias quedó claro el sentir general de los convencionales de que era necesario acometer una profunda reforma de sus estructuras e ir hacia una profundización para hacer que la Unión pudiera afrontar los retos que le exige el actual contexto internacional. Los trabajos de la Convención han puesto de manifiesto una serie de debates y de interrogantes sobre el modelo futuro de la política exterior europea10. La pregunta más relevante - pero que quizás se está olvidando- es la que plantea la misma Declaración de Laeken: «¿Cómo hacer que

9 Eneko LANDABURU, Director General de Relaciones Exteriores de la Unión Europea, El País Domingo, 24 de agosto de 2003, pp. 6 y 7.

10 Francisco ALDECOA LUZURRAGA, El debate sobre Política Exterior Común en la Convención Europea, Documento de Trabajo, Real Instituto Elcano, 15 de enero de 2003, en: http://www.realinstitutoelcano.org. 
la Unión se convierta en un factor de estabilidad y en un modelo en un nuevo mundo multipolar?" 11 . Se trata, en definitiva, del interrogante clave cuya respuesta fundamenta la política de responsabilidad 12 .

La Declaración de Laeken, a través de esa pregunta y otras en el mismo sentido, expone la reflexión sobre la finalidad de la Unión Europea, cuál ha de ser su misión: "Europa debe asumir su responsabilidad en la gestión de la globalización" 13. Según Laeken la misión es, por tanto, jugar papeles importantes en la sociedad internacional de la globalización con el objeto de incidir en ella, erigirse en una alternativa a la misma desde el modelo europeo de sociedad del bienestar y la visión europea de las relaciones internacionales, lo que sólo puede lograr desde la profundización en su propio modelo político, económico y social. En definitiva, el objetivo político de la Unión Europea en el siglo XXI resulta actualizado y pasa de ser el logro de la paz entre europeos a la gestión de la globalización, incorporándose al proyecto europeo la idea de los verdes de la gestión del planeta ${ }^{14}$.

En las primeras sesiones de debate de marzo y abril de 2002, dedicadas a qué se espera de la Unión Europea y a las misiones de la Unión Europea, quedó claro el consenso general de que la Unión debía jugar más papeles en la escena internacional. Posteriormente los dias 11 y 12 de julio de 2002, se celebró la sesión plenaria relativa a la acción exterior de la Unión Europea, de la cual surgió la necesidad de reformar los instrumentos y procedimientos de actuación exterior de forma que la Unión

11 Declaración de Laeken sobre el futuro de Europa, Conclusiones de la Presidencia, Consejo Europeo de Laeken, 14 y 15 de diembre de 2001, en: http://ue.eu.int.

12 Francisco ALDECOA LUZURRAGA, "La Unión Europea en el proceso de globalización. Avances de la Política Exterior europea", Revista de la Unión Europea de la UNED, $n^{\circ} 3$, verano 2002; F. ALDECOA LUZURRAGA, "La política de responsabilidad de. la Unión Europea como potencia civil: una aportación a la Agenda de Paz", Tiempo de Paz, $\mathrm{n}^{\circ}$ 65, verano 2002, pp. 37-59.

13 Un interesante estudio de la Declaración de Laeken desde el punto de vista del papel internacional de la Unión Europea es: Juan Antonio CARRILLO SALCEDO, “EI. futuro de la Unión Europea. Algunas reflexiones sobre el papel de Europa en el mundo contemporáneo a la luz de la Declaración de Laeken", Revista de Occidente, febrero de 2002, pp. 13-27.

14 No podemos olvidar el papel trascendental que ha desempeñado el Partido Verde alemán en la revitalización del proyecto político europeo, tanto en la redacción de la Carta de Derechos Fundamentales de la Unión Europea como el lanzamiento del debate sobre el futuro de la Unión. Véase al respecto: Francisco ALDECOA LUZARRAGA, "La innovación política europea y su dimensión exterior", Política Exterior, $n^{\circ} 91$, enerofebrero 2003. 
fuera capaz de desempeñar los papeles que le corresponden en la escena internacional15.

En el marco de la segunda fase, la de estudio, se decidió la creación de dos Grupos de Trabajo específicos para el estudio de la acción exterior, uno general de acción exterior, $y$ otro dedicado a las cuestiones de defensa. En estos meses resultó de enorme relevancia la propuesta conjunta francoalemana en materia de defensa europea, presentada en noviembre, que prácticamente fue adoptada por el Grupo deTrabajo y, que se encuentra en la base de los importantes progresos que la Constitución Europea lleva a cabo en materia de Politica Europea de Seguridad y Defensa ${ }^{16}$.

Los resultados de los Grupos de Trabajo relativos a la acción exterior han sido muy útiles, han servido de base para los trabajos de la tercera fase de negociación y, por tanto, lo principal de la propuesta del Presidium en materia de acción exterior se inspira en sus recomendaciones 17 . A pesar de que los trabajos ya estaban muy avanzados, la reforma de la Política Exterior Común sufrió un parón en la Convención afectada directamente por la división entre Estados miembros derivada de la crisis de Iraq. Así el Presidente Giscard prefirió que el Presidium no elaborara su primer proyecto de articulado hasta haber consultado personalmente a los Jefes de Estado y de Gobierno sobre esta cuestión, lo que hizo con ocasión del Consejo Europeo de Atenas de 16 de abril de 2003, apartándose del método general de funcionamiento de la Convención.

Con el resultado de este debate, el Presidium hizo pública su propuesta sobre acción exterior el 23 de abril siguiente, recogiendo muchas de las recomendaciones de los Grupos de Trabajo 18. De esta propuesta destacaba la regulación conjunta de todos los ámbitos de la política exterior, la

15 El Presidente de la Convención, Valéry Giscard d’Estaign haciendo el balance de la primera fase, la de escucha, llamaba la atención sobre que el único área en que los convencionales han demandado una ampliación de las competencias ha sido en el ámbito exterior. Valéry GISCARD D'ESTAIGN, "Las últimas noticias sobre la Conveción Europea", El País, 22 de julio de 2002.

16 Véase la Contribución a la Convención de Dominique Villepin y Joschka Fischer, que recoge la propuesta conjunta francoalemana en el ámbito de la política europea de seguridad y defensa. Contribución $n^{\circ} 150$, de 22 de noviembre de 2002 (CONV 422/02).

17 Véase respectivamente: GRUPO DETRABAJO VII, ACCIÓN EXTERIOR, Informe Final, 16 de diciembre de 2002, (CONV 459/02) y GRUPO DE TRABAJO VIII, DEFENSA, Informe Final, 16 de diciembre de 2002, (CONV 461/02).

18 CONVENCIÓN EUROPEA, Proyecto de artículos sobre la acción exterior del Tratado Constitucional, 23 de abril de 2003, (CONV 685/03). 
creación de un Ministro de Asuntos Exteriores y la mayoría cualificada como norma de adopción de decisiones en materia de PESC, cuando existiera propuesta del Ministro. Tras el debate de este proyecto en el seno de la Convención, y a causa de la oposición innegociable del Reino Unido, finalmente el Proyecto de Tratado volvería a la unanimidad como regla general para las decisiones de la PESC.

\section{LA CONSTITUCIÓN EUROPEAY SU MODELO POLÍTICO}

El primer progreso para la Política Exterior Común es que exista una Constitución Europea, que recoja y profundice el modelo político y social europeo. El utilizar el término "Constitución" no resulta inocuo ya que "tiene su propia lógica, su propio peso, exige considerar la realidad europea bajo otro ángulo, el de una democracia que se quiere una comunidad de destino que respeta los profundos valores occidentales» 19 .

El Proyecto de Tratado por el que se instituye una Constitución para Europa recoge en su primera parte, la constitucional, los rasgos definitorios del modelo político europeo (de la "polity») en el más puro lenguaje constitucional, constituyendo esta parte una completa novedad en relación a los antiguos Tratados. Directa y didáctica, con la lectura de estos 59 artículos es suficiente para entender el alcance y el contenido de la Constitución. Esta parte consta de nueve títulos: el I, "De la definición y los objetivos de la Unión"; el II, "De los derechos fundamentales y la ciudadanía de la Unión", el III, «De las competencias de la Unión»; el IV, "De las instituciones de la Unión»; el V, "Del ejercicio de las competencias de la Unión»; el VI, «De la vida democrática de la Unión», el VII, "De las finanzas de la Unión»; el VIII, «De la Unión y su entorno próximo y el IX „De la pertenencia a la Unión»20.

El Proyecto de Tratado Constitucional consolida el modelo del federalismo intergubernamental, que se ha desarrollado en la Unión Europea en los últimos diez años, desde el Tratado de Maastricht de 199221. Este

19 Hartmut MARHOLD, “Grandeur et faiblesses de la Convention européenne. Un bilan provisoire", L'Europe en formation, $\mathrm{n}^{\circ} 3-4,2002$, p.7.

20 Conviene señalar que en este último título se contempla por vez primera en la integración europea la posibilidad de que un Estado miembro abandone voluntariamente la Unión.

21 El concepto de federalismo intergubernamental se debe a dos politólogos franceses, Croisat y Quermonne, que lo conciben como un modelo singular que concibe elementos de integración federal con otros de cooperación entre los Gobiernos para. 
modelo caracterizado por la utilización conjunta de métodos federales de integración y confederales a la vez, se manifestaba en sus orígenes de una forma implícita y se consideraba un mal menor, una fórmula transitoria, que ha ganado carta de naturaleza casi sin darnos cuenta. Con la Constitución se produce una innovación que reside en su aceptación generalizada de una forma explícita como el modelo político propio de Europa, según el cual se va a tratar de llevar a cabo el proyecto político de integración.

El federalismo intergubernamental no es una fórmula intermedia entre posturas federalistas e intergubernamentales, que mezcle algo de las dos lógicas. Se trata, por el contrario, de una innovación, no una nueva fórmula, en la cual se afirman los dos extremos, que contiene elementos de los dos modelos y en el cual se da una cierta división "rationae materiae". El artículo 1 de la Constitución Europea define el modelo político europeo, que recoge con toda exactitud la naturaleza del modelo de federalismo intergubernamental de ciudadanos y Estados $y$, si bien el término federal que constaba en la primera propuesta ha sido sustituido por "comunitario" por la oposición de algunos Estados miembros, en nada queda afectado el modelo político europeo que no se toca22.

La segunda parte del artículo 1 afirma que la Unión se encuentra abierta a todos los Estados europeos que compartan sus valores. Se puede considerar que se trata de una definición de la Unión Europea «hacia fuera", y que tiene la repercusión de establecer una frontera con los paises no europeos. Este artículo, sin embargo, se completa con otros de la Constitución como el Título VIII, que dispone que la Unión establecerá un marco de relaciones privilegiadas basadas en la asociación con su entorno próximo y con el III-193 que dispone que la Unión desarrollará relaciones con terceros países 0 asociaciones internacionales que compartan los mismos valores ${ }^{23}$.

la gestión común de diferentes políticas. Véase al respecto: Maurice CROISAT y JeanLouis QUERMONNE, L'Europe et le fédéralisme, París, Montchrestien, pp. 59-106.

22 El artículo 1.1 establece que: “La presente Constitución, que nace de la voluntad de los ciudadanos y de los Estados de Europa de construir un futuro común, crea la Unión Europea, a la que los Estados miembros confieren competencias para alcanzar sus objetivos comunes. La Unión coordinará las políticas de los Estados miembros encaminadas a lograr dichos objetivos y ejercerá, de modo comunitario, las competencas que éstos le transfieran".

23 El diseño de una política de vencidad para la Unión Europea es otro de los debates que ha trascendido el marco de los trabajos de la Convención. En este sentido, la Comisión Europea presentaba en marzo pasado una Comunicación sobre, una política de vencidad de la Unión Europea, como nuevo marco de relación tras la ampliación: 
La Constitución explicita la naturaleza política del modelo de la Unión al exponer en los primeros artículos los rasgos principales de este peculiar modelo político transnacional. Dando un paso más los cimientos de esa organización, sus valores y objetivos, suponen la asunción y manifestación del modelo europeo de sociedad. En este sentido, el artículo 2 recoge los valores de la Unión, esto es, los valores básicos que hacen que los pueblos europeos se sientan parte de la misma Unión ${ }^{24}$. Son valores, en definitiva, que emanan de la sociedad europea, que son la razón de ser del proyecto europeo y que se completan con la incorporación de la Carta de Derechos Fundamentales de la Unión en la parte segunda 25.

En el artículo 3 se recogen los objetivos de la Unión, que se conciben como los uprincipales fines que hacen necesaria la creación de la Unión para el ejercicio de determinados poderes en común a escala europea» 26 . Se enuncian los objetivos generales que justifican la existencia de la Unión como entidad política y no los objetivos a conseguir a través de diferentes políticas como era el caso de los objetivos en la redacción de los Tratados. Basta la simple comparación entre el artículo 2 del Tratado de la Unión Europea y el artículo 2 del Tratado de la Comunidad Europea con este artículo del Tratado Constitucional para percibir el cambio de óptica existente, el paso del método económico de integración a la integración política.

En los primeros casos los objetivos son más bien los sectoriales o puntuales a perseguir con esa asociación especial, son los objetivos de una organización internacional, aún cuando hagan referencia a determinados valores. En el caso delTratado Constitucional se establecen objetivos generales, que coinciden con los de una organización política de ámbito de

COMISIÓN EUROPEA, “Una Europa más amplia: un nuevo marco para las relaciones con nuestros vecinos del Este y del Sur de Europa", 1 de marzo de 2003, (COM (2003) 104). Para una valoración de este nuevo enfoque, véase:William WALLACE, Looking after the neighbourhood: responsibilities for EU 25, Notre Europe Policy Papers $n^{\circ} 4$, July 2003, en: http.//www.notre-europe.asso.fr.

24 El artículo 2 establece que: "La Unión se fundamenta en los valores de respeto a la dignidad humana, libertad, democracia, igualdad, Estado de Derecho y repeto a los derechos humanos. Estos valores son comunes a los Estados miembros en una sociedad caracterizada por el pluralismo, la tolerancia, la justicia, la solidaridad y la no discriminación"

25 Marhold considera que la constitucionalización de la Carta tiene importantes repercusiones para la acción exterior de la Unión, que quedará vinculada a un conjunto de valores que le dará una legitimidad añadida a la defensa de meros intereses. Hartmut MARHOLD, "Grandeur et faiblesses de la Convention européenne. Un bilan provisoire", op. cit., p. 8.

26 Según la nota explicativa de la segunda parte del documento CONV 528/03. 
actuación general. Son los objetivos de una verdadera politeia que han de guiar todas sus actuaciones en lo económico, en lo político, en lo social y en lo internacional. El cambio reside también en una primera redacción en la "lengua de los Tratados" para pasar al lenguaje constitucional del Tratado Constitucional, se pasa del idioma diplomático de las relaciones entre Estados al idioma de los ciudadanos.

\section{UNIDADY COHERENCIA EN LA POLÍTICA EXTERIOR COMÚN.}

Muchos de los avances políticos y técnicos de la Constitución apuntan a reforzar la unidad y coherencia de la actuación exterior de la Unión, lo que viene ante todo a afirmar el concepto de Política Exterior Común, como el conjunto de la actuación exterior de la Unión. Uno de los primeros consensos a los que llegó la Convención es que la Unión Europea debe contar con una personalidad jurídica, expresa y única, que sustituya a las de las tres Comunidades actualmente existentes, convirtiéndose en sujeto de Derecho internacional27. La creación de una personalidad jurídica única, en el artículo 4, permite la fusión de los distintos Tratados de las Comunidades Europeas, lo que aporta mayor claridad $y$ hace posible su reorganización en un Tratado Constitucional de la Unión Europea28.

Esta decisión presenta consecuencias de enorme relevancia para la dimensión exterior de la Unión Europea y la Política Exterior Común. En este sentido, se ha decidido otorgar a la Unión Europea personalidad jurídica internacional, capacidad para concluir Tratados, derecho de legación activo, competencias explícitas en relaciones exteriores en los casos en que detente competencias implícitas, etc ${ }^{29}$. Duke considera esta decisión como una de las más importante de la Convención y estima que la instau-

27 Hay que llamar la atención sobre que, en la última sesión de la Convención, el 10de julio se decidía finalmente mantener la personalidad jurídica independiente del Euratom, que pasa a regularse en un Protocolo anexo a la Constitución.

28 Jean-Victor Louis llama la atención sobre la atribución de personalidad jurídica a la Unión ha sido uno de los consensos más valiosos de la Convención, que en pocos meses ha podido acordar algo que no fueron capaces de lograr las dos últimas Conferencias Intergubernamentales. Jean-Victor LOUIS, "La Convention et l'avenir de I'Union européenne", Cahiers du droit européen, n³-4, 2002, p. 237.

29 En relación a la personalidad jurídica única de la Unión Europea y su subjetividad internacional, véase: Natividad FERNÁNDEZ SOLA, “La política exterior y la proyección externa de la Unión Europea. Impacto de los trabajos de la Convención Europea", Revista de Estudios Políticos, n 119, enero-marzo 2003, pp. 381-416. 
ración de la personalidad jurídica de la Unión abre por sí sola la posibilidad de que la Unión desarrolle su propio cuerpo diplomático ${ }^{30}$. Igualmente presentará enormes diferencias en lo relativo a la representación de la Unión ante organizaciones internacionales.

Desde otro punto de vista, la decisión sobre la personalidad jurídica única de la Unión tiene como consecuencia la superación de la estructura de pilares, lo que redundará en una mayor unidad y coherencia de la política exterior. Sin embargo, la desaparición de los pilares no significa que todos los ámbitos de la política exterior vayan a ser sometidos a los mismos procedimientos de toma de decisiones. Se mantiene, por el contrario, el modelo intergubernamental para la Política Exterior y de Seguridad Común y para la Política de Defensa, basado en la unanimidad y en el control del Consejo. Se reafirma así la Política Exterior Común basada en el modelo de federalismo intergubernamental, conviviendo métodos federales en los ámbitos comunitarios e intergubernamentales en la PESC y la PESD.

Desde el inicio existió el consenso, que ya se reflejaba en el Anteproyecto deTratado Constitucional de octubre de 2002, de regular conjuntamente en la parte dedicada a las políticas y acciones de la Unión todos los instrumentos de la acción exterior, lo que refuerza la unidad en la concepción de la misma. En este sentido, en la parte III de la Constitución se incluye el Título $\mathrm{V}$ "De la acción exterior de la Unión» donde se regulan en los distintos capítulos las disposiciones de aplicación general; la PESC y la política de defensa; la política comercial común; la cooperación al desarroIlo, la cooperación económica, financiera y técnica con terceros países y la ayuda humanitaria; las medidas restrictivas o sancionadoras; los acuerdos internacionales; las relaciones de la Unión con Organizaciones Internacionales y terceros paises y la nueva cláusula de solidaridad.

Esta regulación unitaria es posible, sobre todo, gracias a la personalidad jurídica única de la Unión Europea y la decisión de eliminar los pilares. Esta medida contribuye a reforzar decisivamente la concepción global y unitaria de la Política Exterior Común, sobre todo, en cuanto que todos. sus ámbitos se verán afectados por los principios y objetivos generales en materia de acción exterior. Otras innovaciones de la Constitución que se estudiarán posteriormente contribuyen igualmente a la unidad y coherencia de la Política Exterior Común, como puede ser el nuevo Ministro de Asuntos Exteriores o el Servicio Europeo de Acción Exterior.

30 Simon DUKE, The Convention, the draft Constitution and the External Relations: Effects and Implications for the EU and its international role, European Institute of Public Administration, September 2003, pp. 7-11, en: http://www.eipa.nl. 


\section{DEL INTERÉS NACIONAL A LA POLÍTICA DE RESPONSABILIDAD.}

De la Política Exterior Común, esto es, de la actuación de la Unión en la última década y del mandato de Laeken ya se atisba cuál ha de ser la presencia de la Unión en la escena internacional, cuál es su modo de entender las relaciones internacionales. Esas características de la concepción europea de la política exterior serían, a nuestro entender, las siguientes: la dimensión externa de los valores de la Unión, la regulación, la cooperación multilateral, la dimensión externa del modelo social europeo y la exportación del modelo europeo de estabilidad.

Estas características demuestran que existe una concepción común, uniforme y progresiva de las relaciones internacionales por parte de todos los miembros de la Unión, que es consecuencia del modelo social de bienestar común, esto es, responde a valores compartidos, intereses comunes y una misma visión del mundo. Todas ellas han sido plasmadas de una u otra manera en la definición de principios y objetivos de la acción exterior de la Unión Europea, tanto en el artículo 1-3 como en el III-193.

Los principios y objetivos de la actuación exterior europea no sólo se contemplan en la parte segunda de la Constitución donde se desarrolla la acción exterior europea, sino también en el artículo 3 de la Parte Primera, constituyendo uno de los cuatro objetivos de la Unión Europea, esto es, los que le dan su razón de ser. El artículo 3.4 establece que «En sus relaciones con el resto del mundo, la Unión afirmará y promoverá sus valores e intereses. Contribuirá a la paz, la seguridad, el desarrollo sostenible del planeta, la solidaridad y el respeto mutuo entre los pueblos, el comercio libre y equitativo, la erradicación de la pobreza y la protección de los derechos humanos, especialmente los derechos del niño, la estricta observancia y el desarrollo del Derecho Internacional, y en particular el respeto a los principios de la Carta de las Naciones Unidas".

Por tanto, los principios de la acción exterior europea se consagran en el nivel constitucional, y no sólo eso sino que en uno de los artículos nucleares, cimiento de toda la Constitución. En esta formulación se recogen en buena medida las características de la actuación exterior de la Unión, que hemos establecido previamente, y responde a una concepción global de la actuación exterior no únicamente centrada en la consecución de los intereses europeos, sino que responde, en gran medida, a una "ética» o concepción de una política exterior basada en los valores ${ }^{31}$. En

31 Hazel Smith estudia el papel que tienen los valores - lo que ella llama la ética "ethics" en la política exterior europea. Considera que, si bien en algunas ocasiones los 
este sentido, se manifiestan especialmente en el compromiso europeo con la promoción de la paz y los valores europeos, la gestión de la globalización, unas relaciones internacionales justas y la regulación como marco de desarrollo de las relaciones entre Estados. Se trata en buena parte de una redacción inspirada en la política de responsabilidad.

En el mismo sentido, en el artículo III-193 dedicado a la acción exterior, recoge y desarrolla más detalladamente los principios y valores de la acción exterior europea que, en definitiva, son los que deben inspirar la actuación de la Unión. Este artículo sigue fielmente la redacción de principios y objetivos de la acción exterior propuesta por el Grupo VII en su informe. En el párrafo primero se indica que la acción de la Unión Europea se basará en los principios que han llevado a su creación. Se recogen principalmente el fomento de los valores europeos en el mundo: democracia, derechos humanos, Estado de Derecho, dignidad humana, igualdad y solidaridad, estos dos últimos, característicos de la construcción europea.

A ellos se les añaden los principios específicos de la acción exterior como es el respeto del Derecho internacional conforme a la Carta de Naciones Unidas, el establecimiento de relaciones $y$ asociaciones con Estados o grupos de Estados que compartan los mismos valores y el fomento de soluciones multilaterales a los problemas internacionales, con preferencia en el marco de Naciones Unidas.

En el párrafo 2 del artículo III-193 se recogen explícitamente los objetivos de la actuación exterior europea. Establece muchas novedades respecto a la anterior redacción en el artículo 11 del Tratado de la Unión Europea, en cuanto que asume el acervo consolidado de los objetivos europeos en la escena internacional en los apartados a, b y $c_{r}$ pero introduce algunas modificaciones de interés, como es sustituir la expresión "comunes" por de "la Unión», en relación a los valores, intereses, etc. Esto pone de manifiesto que se pretende reforzar la dimensión de Unión en detrimento de la actuación conjunta de los Estados miembros. La otra adición llamativa es que en el párrafo $b$ de apoyo y consolidación de la democracia, el Estado de Derecho, los derechos humanos, se añade ahora el Derecho internacional. Mención que no puede resultar inocente a resultas de la pasada crisis de Iraq, y que refuerza el compromiso europeo con la regulación internacional como fórmula de gestión de las relaciones internacionales.

intereses predominan, como norma general se trata de una política exterior concebida desde la ética por la presión ejercida por la sociedad europea. Véase Hazel SMITH. European Union Foreign Policy, London, Pluto Press, 2002, 270-272. 
Los siguientes apartados, $d$ al $h$, son absolutamente novedosos, no encontrando un precedente en el Tratado de la Unión Europea. Se desarroIlan explícitamente elementos de la denominada política de responsabilidad en relación a distintos aspectos. Así el apartado d establece el objetivo de fomentar un desarrollo económico y sostenible de los países en vías de desarrollo para erradicar la pobreza. En el se propone estimular la integración de todos los paises en la economía mundial incluso a través de la abolición de las restricciones al comercio internacional.

En el $f$ manifiesta su compromiso con la protección del medio ambiente y los recursos naturales internacionales $y$ el desarrollo sostenible; en el g se recoge la misión de ayudar a las poblaciones que se enfrenten a catástrofes naturales o producidas por el hombre. Finalmente, en el punto $h$ se reitera el compromiso europeo con un sistema internacional "basado en una cooperación multilateral sólida y el buen gobierno a escala mundial». Se está planteando la lógica de la gestión del planeta basada en marcos multilaterales y fórmulas de gobernabilidad mundial, características del modelo europeo para la gestión de la globalización.

Existe la firme voluntad de que estos objetivos no queden en una mera declaración de intenciones y así la Convención ha debatido la posibilidad de introducir bases jurídicas nuevas para poder llevarlos a cabo. En este sentido, se ha incluido un nuevo artículo, el III-222, que permite a la Unión prestar ayuda financiera a las balanzas de pagos de terceros países cuando se requiera una acción urgente. Igualmente se ha creado la base jurídica necesaria en el III-223 para que la Unión preste ayuda humanitaria de urgencia, desgajándola de la cooperación al desarrollo, ya que tiene un carácter específico frente a ésta, no pudiendo resultar condicionada ni a cláusulas políticas ni de otra clase.

En definitiva, con esta ambiciosa declaración de principios y objetivos se está proyectando la voluntad de cambiar el sistema mundial con la finalidad de hacer la globalización gobernable, recogiendo con éxito el guante lanzado por la Declaración de Laeken. De la comparación de los actuales objetivos de la Política Exterior, regulados en el Tratado de la Unión Europea con la nueva propuesta de redacción se percibe un salto cualitativo, pasándose de una concepción de la política exterior europea basada en el interés común a una formulación explícita de la política de responsabilidad. 


\section{EL MINISTRO DE ASUNTOS EXTERIORES: LA CARA DE LA POLITICA EXTERIOR COMÚN.}

La innovación institucional más relevante del Tratado Constitucional la constituye la creación del Ministro de Asuntos Exteriores, con la doble dimensión de ser miembro de la Comisión, y uno de sus Vicepresidentes, y mandatado por el Consejo. El artículo 27 establece que será nombrado por mayoría cualificada por el Consejo Europeo, con la aprobación del Presidente de la Comisión. El Ministro se encontrará al frente de la Política Exterior y de Seguridad Común, incluida la defensa. Contribuirá con sus propuestas a la formulación de ambas y las ejecutará como mandatario del Consejo.

En su dimensión de miembro de la Comisión, el Ministro de Asuntos Exteriores es a la vez un Vicepresidente de la misma, encargado de las relaciones exteriores y de la coordinación de todos los aspectos de la acción exterior de la Unión. El artículo 27 prosigue añadiendo que: «En el ejercicio de estas responsabilidades dentro de la Comisión, y exclusivamente por lo que respecta a las mismas, el Ministro de Asuntos Exteriores de la Unión estará sujeto a los procedimientos por los que se rige el funcionamiento de la Comisión". Parece, por tanto, que en cuanto comisario participa en la colegialidad de la Comisión, está bajo el mandato de su Presidente y se encuentra sujeto al control del Parlamento Europeo, control que no podrá verificarse en el caso de la PESC. El artículo 23 añade que en su dimensión intergubernamental será el encargado de presidir el Consejo de Asuntos Exteriores de la Unión.

El Ministro de Asuntos Exteriores presenta una doble dimensión, y por tanto, podrá formular iniciativas en materia de PESC propias y defenderá las iniciativas de la Comisión en las cuestiones relativas a las relaciones exteriores. Dirigirá la unidad de política exterior y las Delegaciones de la Unión, actuales Delegaciones de la Comisión, también quedarán bajo su autoridad. El artículo 39.4 igualmente le encomienda la ejecución de la Política Exterior y de Seguridad Común junto a los Estados miembros. Por tanto, parece que los actuales responsables de la ejecución de la PESC - Presidencia, troika, Secretario General del Consejo y Comisiónson sustituidos por el Ministro de Asuntos Exteriores, simplificándose considerablemente las responsabilidades.

Tanto el Parlamento Europeo como la Comisión coinciden en apreciar la creación de este Ministro que, a su juicio, redundará en la coordinación y en la visibilidad de la actuación exterior europea. Ambos insisten en la necesidad de que para que pueda actuar con eficacia es esencial que el Ministro pueda apoyarse en una única administración -el Servicio 
Exterior Europeo- que radique en, $o$ al menos esté vinculada a, la Comisión 32 . En su Comunicación sobre el proyecto deTratado, la Comisión introduce una importante novedad al anunciar la posibilidad de que la futura Comisión se organice por Grupos de comisarios. Entonces el Ministro de Asuntos Exteriores presidiría el grupo encargado de las relaciones exteriores, para garantizar la coordinación del conjunto de la acción exterior. Parece que presidiendo, a la vez, el Grupo de comisarios y el Consejo de Asuntos Exteriores puede efectivamente encontrarse la coherencia y la eficacia exterior de la Unión.

Sin embargo, los analistas no coinciden en apreciar positivamente esta novedad. Así algunos estiman que esta reforma no basta para crear una política exterior frente a la resistencia de los Estados miembros a ceder soberanía y que será un Ministro sin política que aplicar ${ }^{33}$. Otros ven problemático el "servir a dos señores" y la relación con el recién creado Presidente del Consejo Europeo, el Presidente de la Comisión así como con los Ministros de Asuntos Exteriores nacionales, con los que frecuentemente chocará34. En este punto, cabe plantearse por su carácter político, si tenderá a integrarse en la Comisión y, en este sentido, "comunitarizar" la práctica de la PESC o, por el contrario, se tratará de un "submarino" intergubernamental colado dentro de la Comisión. En cualquier caso, parece que esta innovación puede otorgar a la dirección de la Política Exterior Común el perfil político necesario, adecuado a su carácter de «alta política».

\section{LATOMA DE DECISIONES EN LA POLÍTICA EXTERIOR Y DE SEGURIDAD COMÚN}

La cuestión del mantenimiento de la unanimidad $y$ el veto en la Política Exterior y de Seguridad Común es, a nuestro juicio, el mayor

32 Véase respectivamente: PARLAMENTO EUROPEO (Comisión de Asuntos Constitucionales), Informe sobre el proyecto de Tratado por el que se instituye una Constitución para Europa y dictamen del Parlamento Europeo sobre la convocatoria de la Conferencia Intergubernamental, 9 de septiembre de 2003, (2003/0902(CNS)), punto 21; y COMMISSION DES COMMUNAUTÉS EUROPÉENNES, Communication de la Commission "Une Constitution pour I'Union", 17 de septiembre de 2003, (COM(2003)548), pp. 11-12.

33 Kirsty HUGHES, A Dinamic and Democratic EU or Muddling through Again? Assessing the EU's Draft Constitution, EPIN Working Paper $n^{\circ}$ 8, July 2003, pp. 10-11.

34 Hartmut MARHOLD, "Grandeur et faiblesses de la Convention européenne. Un bilan provisoire", op. cit., p. 10, Simon DUKE, The Convention, the draft Constitution and the External Relations: Effects and Implications for the EU and its international role, op. cit., pp. 14-17. 
obstáculo de la Política Exterior Común que la Convención no ha sido capaz de superar. Conseguir decisiones en cuestiones tan sensibles como la política exterior a veinticinco parece prácticamente imposible. Es, en definitiva, condenar la Unión a la inacción. Tanto el Grupo de Trabajo de Acción Exterior como la primera propuesta de redacción contemplaban fórmulas que permitían introducir mayorias cualificadas o supercualificadas en determinados casos. La mayoría cualificada en la PESC también había sido expresamente apoyada por Francia y Alemania en su contribución conjunta sobre la arquitectura institucional35. Sin embargo, por el veto expreso del Reino Unido, finalmente se volvía a la unanimidad como regla general de decisión en el artículo 39.7.

El mantenimiento de la unanimidad, sin embargo, se rodea de disposiciones que pretenden atenuarla. Así en el artículo 39.8. se regula una pasarela de la unanimidad a la mayoría cualificada como excepción a la pasarela general del artículo 24.4. Para la PESC se dispone que el Consejo Europeo por unanimidad podrá aprobar que el Consejo decida por mayoría cualificada en un ámbito concreto. En este caso, parece que no rige la obligación de informar previamente a los Parlamentos Nacionales ni al Parlamento Europeo.

La otra disposición de atenuación de la unanimidad es la flexibilización en el establecimiento de cooperaciones reforzadas tanto en materia de política exterior como en defensa. Parece que si una decisión o una política no puede ponerse en marcha en este ámbito por mayoría cualificada, podría recurrirse a una cooperación reforzada, siempre que se contara con un tercio de los Estados miembros, en el seno de la cual, los participantes pueden decidir que las cuestiones que según el Tratado se adoptan por unanimidad lo hagan por mayoría cualificada.

Los analistas coinciden en que más que innovaciones políticas lo que realmente necesita la PESC es el abandono de la unanimidad y hasta que no se consiga siempre se habrá avanzado poco ${ }^{36}$. De la misma manera, el Parlamento Europeo y la Comisión lamentan los pocos avances en este

35 CONVENCIÓN EUROPEA, Contribución de Dominique de Villepin y Joschka Fischer sobre la arquitectura institucional, Contribución $n^{\circ} 192,16$ de enero de 2003, (CONV 489/03).

36 Kirsty HUGHES, A Dinamic and Democratic EU or Muddling through Again?..., op. cit., p. 11; Hartmut MARHOLD, "Grandeur et faiblesses de la Convention européenne. Un bilan provisoire", op. cit., p. 12; Simon DUKE, The Convention, the draft Constitution and the External Relations: Effects and Implications for the EU and its international role, op. cit., p. 32; Steven EVERTS y Daniel KEOHANE, The European Convention and EU Foreign Policy: Learning from Failure, op. cit., p.6. 
campo. Así el Parlamento Europeo considera un defecto del Proyecto de Constitución que no se contemple "la supresión de la unanimidad requerida en el Consejo en algunos sectores vitales" y que "debería sustituirse la unanimidad, en los casos en que se mantiene, a más tardar antes del 1 de noviembre de 2009, por una mayoría supercualificada que represente tres cuartas partes de los Estados miembros y de la población de la Unión ${ }^{37}$.

La Comisión, a su vez, reflexiona sobre que el mantenimiento del veto nacional es equivalente a condenar a la Unión a la imposibilidad de cumplir los objetivos que el Tratado Constitucional le impone. Propone que se consideren en el seno de la CIG alternativas a la unanimidad y el veto, como considerar mayorías cualificadas reforzadas o una nueva definición de la unanimidad, según la cual después de un cierto periodo de debate en el seno del Consejo y una discusión en el Consejo Europeo, la oposición de uno o dos Estados no pueda impedir la adopción de la decisión 38 .

\section{PASOS HACIA LA DIPLOMACIA COMÚN EUROPEA EN LA CONSTITUCIÓN}

En los últimos días de trabajo los convencionales han mejorado sustancialmente el texto en muchas cuestiones, entre las que destaca la de la diplomacia común europea. Como es sabido, la Unión Europea no cuenta con un servicio diplomático propio, que ejecute su política exterior, sino que se apoya tanto en las Delegaciones que la Comisión tiene ante terceros Estados como en las propias Embajadas de los Estados miembros, lo que produce algunas incoherencias y solapamientos.

En el año 2000 el Parlamento Europeo aprobó un Informe pionero en el que se pedía la constitución de una diplomacia común europea y de una escuela diplomática europea, encargada de la formación del cuerpo diplomático europeo y de la preparación de los diplomáticos nacionales ${ }^{39}$. Lo cierto es que, salvo algunas iniciativas aisladas, esta importante cuestión

37 PARLAMENTO EUROPEO (Comisión de Asuntos Constitucionales), Informe sobre el proyecto de Tratado por el que se instituye una Constitución para Europa y dictamen del Parlamento Europeo sobre la convocatoria de la Conferencia Intergubernamental, op. cit., puntos 9 y 24.

38 COMMISSION DES COMMUNAUTÉS EUROPÉENNES, Communication de la Commission "Une Constitution pour I'Union", op. cit., pp. 7-8.

39 PARLAMENTO EUROPEO, Resolución sobre el establecimiento de una diplomacia común para la Comunidad Europea, 5 de septiembre de 2000, (A5-0210/2000). 
no ha recibido el seguimiento que su relevancia merecía por parte de las instituciones europeas hasta la Convención.

En los trabajos de la Convención la cuestión de la constitución de un servicio exterior de la Unión ha recibido una importante atención. El Grupo de Trabajo de Acción Exterior llegaba a recomendar la creación de una escuela diplomática así como de un servicio diplomático propio de la Unión Europea, similar al de los Estados miembros y la redenominación de las delegaciones de la Comisión como Embajadas de la Unión. Sin embargo, a lo largo de los diversos debates en el pleno, esta cuestión se reveló como altamente delicada y recibió la oposición frontal de muchos de los Estados miembros, con el Reino Unido a la cabeza. Los recelos no son de extrañar, dado que la diplomacia es una de las cuestiones que quizás más se identifican con la soberanía del Estado.

La sorpresa ha venido de la mano de la última versión de la Constitución Europea, aprobada.por consenso de todos los convencionales el 10 de julio pasado. El artículo III- 197 alude explícitamente a la creación de un Servicio Europeo de Acción Exterior, que trabajará en colaboración como los servicios diplomáticos de los Estados miembros. Este servicio exterior, del cual saldría el personal de las delegaciones de la Unión, quedaría bajo la autoridad directa del Ministro de Asuntos Exteriores de la Unión. Esa disposición se completa con una Declaración en que la Convención recomienda que ese servicio exterior esté compuesto por personal competente de la Comisión, del Consejo y de los servicios diplomáticos nacionales de los Estados miembros 40 .

La idea que la Constitución tiene de Servicio Europeo de Acción Exterior responde al concepto de diplomacia común europea, que se entiende como compuesta tanto por miembros de las instituciones comunes como de los Estados miembros y asocia en estrecha cooperación a las instituciones comunes con las administraciones nacionales ${ }^{41}$. Este instrumento, de naturaleza federal, es el más adecuado para la ejecución de una política exterior europea que también es común y no única. Por el contrario, la diplomacia europea única sería la que tendería a reemplazar las diplomacias nacionales por un único cuerpo europeo.

40 CONVENCIÓN EUROPEA, Declaración sobre la creación de un Servicio Europeo de Acción Exterior, anexa al proyecto de Tratado por el que se instituye una Constitución para Europa, 13 de junio y 10 de julio de 2003, (CONV 850/03), p. 239.

41 Simon DUKE, "Preparing for European diplomacy?", Journal of Common Market Studies, Vol. 40, ${ }^{\circ}$ 5, 2002, pp. 849-870. 
El concepto de diplomacia común europea pone el énfasis en el desarrollo de una cultura diplomática europea entre todos sus agentes así como una lógica de la cooperación mutua y la complementariedad entre el cuerpo europeo y las diplomacias de los Estados miembros 42 . En este sentido, el ideal de futuro es encontrar al prototipo de diplomático europeo tanto en el Servicio Exterior de la Unión como en cualquier representación de un Estado miembro, con la misión todos ellos de desarrollar lo valores, los intereses y la visión del mundo europeos ante terceros y en foros internacionales.

La Unión Europea tiene ante si hoy el reto de convertirse en líder de la sociedad internacional de la globalización. Indudablemente la diplomacia común europea es uno de los instrumentos claves para lograr este objetivo, más incluso que un ejército, otro de los nuevos avances. La Convención así lo ha entendido y, por ello, ha tomado las medidas pertinentes en la Constitución Europea. Ahora se deja en manos de los Estados miembros que tomen las decisiones adecuadas para su puesta en marcha, para lo que la Convención da un año desde su entrada en vigor.

\section{LA POLÍTICA EUROPEA DE SEGURIDADY DEFENSA: LA GEOMETRIAA VARIABLE}

Indudablemente el avance de altura lo ha experimentado el área de la defensa europea donde son muchas y de gran calado las novedades introducidas. La nueva voluntad política de que Europa juegue papeles en la sociedad internacional de la globalización se vislumbra en una nueva concepción: hasta ahora era una intervención exterior y no una típica defensa, y ahora, junto a la defensa existente como medio de ayuda a terceros se pasa a configurar instrumentos que posibiliten la defensa futura de la Unión Europea. La fórmula que ha hecho posible avanzar en la articulación de la defensa europea es la flexibilidad, que permitirá a cada uno

42 Hemos estudiado en otro lugar la importancia de la formación como paso para la constitución de una Diplomacia Común Europea. Véase al respecto: Francisco ALDECOA LUZARRAGA, La coordinación de la formación en materia comunitaria de los cuerpos diplomáticos de los Estados miembros, Encuentro de Escuelas e Institudos Diplomáticos Europeos, Madrid 20y 21 de mayo de 2002. Se recogen expresamente algunas de nuestras propuestas en la contribución de Méndez de Vigo a los trabajos del Grupo VII. Véase: Contribución Íñigo Méndez de Vigo al Grupo de Trabajo VII, "Hacia el establecimiento de una diplomacia común europea", 3 de diciembre de 2002, (WG VIIWD 55). 
suscribir sus compromisos"como desee, aunque presente el inconveniente de la complejidad.

La política exterior europea se articula en torno a la noción de potencia civil ${ }^{43}$. La disyuntiva sobre si la Unión Europea debe convertirse en una potencia militar o una potencia civil es uno de los debates que ha presidido los trabajos de la Convención, debate reforzado por la crisis de Iraq y la percepción del escaso relieve europeo frente a la hegemonía militar de Estados Unidos 44 . La cuestión es si la Unión Europea puede seguir siendo una potencia civil aunque se dote de instrumentos militares y si puede concebirse una política de defensa a pesar de la existencia de distintas percepciones entre los países de la Unión 45.

La UE actual es ya muy amplia y heterogénea, con diversas maneras de interpretar las demandas de seguridad y defensa por parte de los distintos Estados: los que celebraron en abril pasado la Minicumbre en materia de defensa y que han mostrado su determinación de crear una Unión Europea de Seguridad y Defensa46, los neutrales, los que privilegian la OTAN, el Reino Unido con una especial relación con Estados Unidos, los

43 R.G.WHITMAN, From Civilan Power to Superpower?The International Identity of the European Union London, Macmillan, 1998.

44 Desde el punto de vista doctrinal se ha hecho famoso en los últimos meses el ensayo del politólogo norteamericano Robert Kagan, que reflexiona sobre las diferencias entre Estados Unidos y Europa en la propia concepción de su política exterior y, sobre todo, de las necesidades de defensa. Robert KAGAN, "Power and Weakness", Policy Review, $\mathrm{n}^{\circ} 113$, June 2002, en: http://.policyreview.org. En dirección distinta véanse los interesantes análisis de: Joseph NYE, La paradoja del poder norteamericano, Madrid, Taurus, 2003; Emmanuel TODD, Después del imperio, Madrid, Foca, 2003.

45 Un interesante artículo estudia si la creación de una Política de Defensa europea hace que el concepto de potencia civil aplicado a la Unión Europea se haya convertido en obsoleto. Por el contrario, llega a la conclusión de que la vertiente militar puede fortalecer su carácter de potencia civil y reflexiona sobre la necesidad de diseñar los rasgos de esa potencia civil. Stelios STAVRIDIS, "Militarising" the EU: the concept of Civilian Power Revisited", The International Spectator, $n^{\circ} 4,2001$, pp. 43-50.

46 Francia, Alemania, Bélgica y Luxemburgo celebraran el 29 de abril de 2003 una Cumbre con el objetivo de estudiar la creación de una cooperación reforzada enter ellos para acelerar el establecimiento de una defensa autónoma europea. En su Declaración Final anunciaban su intención de constituir puntual de diferentes objetivos, cooperación abierta a los demás Estados miembros que quieran sumarse. Véase al respecto: Declaración de Bruselas tras la reunión de Jefes de Estado y de Gobierno de Alemania, Francia, Luxemburgo y Bélgica sobre la defensa europea, 29 de abril de 2003, en: http://www.elysse.fr. Resulta clara la interacción entre la Convención y la Minicumbre en materia de defensa de 29 de abril, estando la Constitución preparada para acoger en su seno esta cooperación reforzada, que denomina "cooperación estructurada" e incluso, para contar con sus capacidades como instrumento europeo. 
Estados pequeños y los que no conciben una defensa más allá del Estado. Esta heterogeneidad de planteamientos y el que no haya posturas cerradas es lo que ha permitido el avance.

La Convención ha logrado lo que parecía imposible: conjugar las distintas sensibilidades para diseñar fórmulas que se adapten a cada caso. Asi, entre los puntos de consenso común, aplicables a todos los Estados, cabe citar el incremento de las tareas de Petersberg a un mayor número de supuestos. Por otro lado, se establece en el artículo 42 la muy relevante cláusula de solidaridad entre Estados miembros para el caso de que un Estado reciba un ataque terrorista o sufra una catástrofe natural o de origen humano.

La profundización en el diseño de una defensa propia se hace, por el contrario, según fórmulas flexibles: cooperación estructurada, cooperación más estrecha, cooperación reforzada, etc. La figura de la cooperación estructurada se prevé para los Estados que quieran adquirir compromisos más elevados en materia de capacidades militares, esto es, los que ya han manifestado su interés en crear una Unión Europea de Seguridad y Defensa (art. 40.6). La propia Constitución prevé ya que la Conferencia Intergubernamental incluya en un Protocolo adjunto la lista de los participantes.

El Tratado también crea la cooperación más estrecha para la defensa mutua entre los Estados que voluntariamente deseen asumirla (art. 40.7). Se trata, en definitiva, de la asunción del artículo $V$ de la UEO, equivalente a la cláusula de defensa mutua de la Alianza Atlántica. Se abre igualmente la posibilidad de establecer cooperaciones reforzadas en otros aspectos de la defensa. Por último, se ha previsto una Agencia de Armamento, Investigación y Capacidades Militares, en la cual participarán libremente los Estados que deseen hacerlo (art. 40.3). Se quiere así responder al desafío del desarrollo tecnológico ya que sin independencia tecnológica es imposible la independencia defensiva 47 .

47 Este impulso constitucional no puede mirarse como un hecho aislado sino que es el reflejo - $y$ un paso más - del conjunto de iniciativas que han tenido lugar a lo largo de los últimos meses para desarrollar la defensa europea. Cabe citar la adquisición de capacidades militares por la UE - aviones Airbus-, la puesta en marcha del proyecto Galileo o el lanzamiento del Programa Arianne 5, entre otros. Todo ello no muestra sino la voluntad decidida de desarrollar una defensa propia, aunque el precio a pagar sea la geometria variable. 


\section{LA POLÍTICA EXTERIOR COMÚN, EXPRESIÓN DEL FEDERALISMO INTERGUBERNAMENTAL}

La Política Exterior Común, tal y como se contempla en el Proyecto de Tratado Constitucional es el ámbito que mejor responde al modelo de federalismo intergubernamental, ya que combina en su formulación y decisión procedimientos federales con otros intergubernamentales. En la medida en que el Proyecto de Constitución Europea explicita este modelo y lo fortalece, así también otorga la posibilidad a la Unión de llevar a cabo una Política Exterior Común, más coherente y eficaz.

Por tanto, en primer lugar, la Política Exterior Común va a avanzar porque hay Constitución. En consecuencia, como sostiene Habermas, a «más Europan, esto es, una politeia más desarrollada, más posibilidades de presencia exterior 48 . Además hay que estimar los avances concretos de la reforma de la Política Exterior Común en la Constitución, que ponen a disposición de la Unión los instrumentos y los procedimientos para que la Política Exterior Común se formule y se aplique de una forma más coherente y eficiente. No son sólo retoques técnicos sino una verdadera reforma constitucional en la medida en que se establece una concepción concreta de la política exterior, a través de unos principios y objetivos así como medios e instrumentos para la eficacia de esa política exterior común. Se avanza en la línea de la politica exterior reforzada y se racionaliza y mejora en su conjunto, por lo que se puede hablar de progreso político y perfeccionamientos técnicos. Además será necesario tener en cuenta otros progresos derivados de las sinergias del conjunto del proceso y las interacciones de las distintas reformas.

Si bien es cierto que no se ha avanzado todo lo que se podría, sobre todo en lo relativo a la toma de decisiones por mayoría cualificada, se han dado pasos muy importantes para garantizar la formulación de una política exterior eficiente. Del mismo modo la profundización decisiva en la defensa europea, a través de fórmulas imaginativas, es un resultado que no parecía anticipable al comienzo de los trabajos de la Convención 49 . Quizás la dificultad de alcanzar consensos en esta área muestra como la Política Exterior Común es uno de los ámbitos donde más claramente se aprecia

48 Jürgen HABERMAS, "Por qué Europa necesita una Constitución", New Left Review, $\mathrm{n}^{\circ} 11$, nov-dic. 2001, p. 12.

49 El acuerdo de profundizar en la defensa según el interés y las posibilidades de los distintos Estados miembros llevan a que Landaburu estime que en poco tiempo podriamos estar en presencia de una política europea a dos velocidades. Eneko LANDABURU, Director General de Relaciones Exteriores de la Unión Europea, El País Domingo, 24 de agosto de 2003, pp. 6 y 7. 
que el Tratado Constitucional es el punto de encuentro de posturas encontradas, de federalistas e intergubernamentalistas $y$, ante todo, el punto de partida imprescindible para poder seguir avanzando posteriormente.

Tanto el Parlamento Europeo como la Comisión, en sus informes respectivos sobre los resultados de la Convención Europea y la próxima Conferencia Intergubernamental, valoran en general muy positivamente el proyecto de Tratado Constitucional y el modelo político introducido. En este sentido, el Parlamento estima que se trata de un texto que "expresa la voluntad política de los ciudadanos europeos y de los Estados miembros de una manera solemne y plena» e "insta a la CIG a que respete el consenso logrado por la Convención, evite negociar las soluciones que la Convención obtuvo mediante un equilibrio sutil y a que apruebe sin cambios sustanciales el Proyecto de Tratado....50. La Comisión, por su parte, «es de la opinión de que el proyecto de tratado que instituye una Constitución para Europa debe constituir la base para los trabajos de la $\mathrm{ClG}_{2} 51$.

En relación a la valoración de la reforma global de la política exterior común, el Parlamento Europeo la tiene en consideración, estimando especialmente el fin de la estructura de pilares, la introducción de los valores de la Unión y la creación del puesto del Ministro de Asuntos Exteriores. Lamenta, sin embargo, dos aspectos en que a su juicio no ha habido progresos suficientes, la sustitución de la unanimidad por mayorías, aunque fueran supercualificadas y la falta de control parlamentario de la Política Exterior y de Seguridad Común y la Política Europea de Seguridad y Defensa.

La Comisión, por su parte, en relación a la introducción de nuevos medios de actuación para la Unión en la vertiente exterior considera especialmente relevante la creación del Ministro de Asuntos Exteriores de la Unión, que permitirá desarrollar a la Unión una acción exterior y una representación exterior más coherentes y eficaces, la renovación de las disposiciones de la PESC y el desarrollo de la Política Europea de Seguridad y Defensa Común que permite a los Estados miembros que lo deseen mejorar sus capacidades de acción dentro de un marco común ${ }^{52}$.

50 PARLAMENTO EUROPEO (Comisión de Asuntos Constitucionales), Informe sobre el proyecto de Tratado por el que se instituye una Constitución para Europa, op. cit., puntos 9 y 30 .

51 COMMISSION DES COMMUNAUTÉS EUROPÉENNES, Communication de la Commission "Une Constitution pour I'Union", op. cit., p. 3.

52. COMMISSION DES COMMUNAUTÉS EUROPÉENNES, Communication de la Commission "Une Constitution pour I'Union", op. cit., p. 3. 
A medida que se acerca el comienzo de la Conferencia Intergubernamental, se van haciendo públicas las posiciones de los principales actores de la $\mathrm{CIG}$ en relación al Proyecto de Tratado que han de adoptar o enmendar formalmente. Contra todo lo anunciado por la prensa, la mayor parte de ellos se muestran conformes con el acuerdo de la Convención. Si bien es cierto que algunos Estados o la Comisión plantean demandas de reforma de puntos concretos del Proyecto de Tratado, ninguno rechaza en bloque el progreso de la Convención 53 .

Los Estados fundadores, con la Presidencia italiana a la cabeza y el Parlamento Europeo, respaldan al cien por cien el contenido del acuerdo, afirmando que lo adoptarán íntegramente. Recientemente, ha sorprendido que el Libro Blanco del gobierno británico ante la CIG es más una defensa ardiente de la Constitución de cara a su ciudadanía que el cuestionamiento de los progresos de la Convención 54 . Por todo ello, parece que sin pecar de demasiado optimismo, se puede esperar una Conferencia Intergubernamental rápida que acabe sus trabajos en diciembre en Roma y que suscriba, en lo sustancial el proyecto de Tratado Constitucional. Entonces, en diciembre coincidirá la agenda política -la Estrategia de Seguridad - y los medios técnicos para que Europa pueda enfrentarse con éxito a las nuevas demandas internacionales $y$ asumir sus responsabilidades en la gestión del planeta.

53 Así a título de ejemplo, la Comisión y algunos países pequeños solicitan la modificación de las nuevas reglas de la composición de la Comisión o España y Polonia demandan una revisión del acuerdo sobre la mayoría cualificada.

54 Véase al respecto el Libro Blanco del gobierno británico ante la CIG: SECRETARY OF STATE FOR FOREIGN AND COMMONWEALTH AFFAIRS, A ConstitutionalTreaty for the EU. The British Approach to the European Union Intergovernmental Conference, September 2003. 